\title{
Multiphysics simulation of a solid oxide fuel cell based on COMSOL method
}

\author{
QIAO Yaoxuan ${ }^{1}$, FAN Cheng $^{1 *}$, SUN Kening $^{1 *}$ \\ ${ }^{1}$ College of Chemistry and Chemical Engineering, Beijing Institute of Technology, Beijing 100081, China;
}

\begin{abstract}
Solid oxide fuel cells (SOFCs) are promising high-effective energy conversion devices for wide fuel sources and high energy efficiency. Based on non-linear kinetics at triple-phase-boundary, a threedimensional model with a single flow channel was constructed. Distribution of mass flow, temperature and current density in a different configuration and working conditions were investigated. Critical factors in voltage output, power output, and reactant utilizations were determined. It is concluded that increasing temperature can give better performances. The increase of inlet flow results in an increased of power density but a decrease of fuel utilization efficiency. The numerical simulation provides a scientific basis for control strategy and structural design of SOFC.
\end{abstract}

\section{Background}

The solid oxide fuel cell (SOFC) can directly convert chemical energy into electrical energy. It has the advantages of clean, high efficiency, high energy density, and good fuel universality. It has unique advantages among many fuel cells ${ }^{[1]}$. Since the solid oxide fuel cell is an all-solid structure, its configuration design is very flexible. At present, there are roughly the following types: flat $\mathrm{SOFC}^{[2]}$, round tube $\mathrm{SOFC}^{[3]}$, flat tube $\mathrm{SOFC}^{[4]}$, Corrugated $\mathrm{SOFC}^{[5]}$ and so on. The flat tube fuel cell combines the advantages of tube and plate fuel cells, which not only effectively shortens the current transmission path, improves the low power density of the tubular design, reduces internal ohmic losses, and is easy to seal, which is of great research and application significance $^{[6]}$.

The electrochemical reaction to SOFC occurs to the interface of the gas phase, the electronically conductive phase and the ionically conductive phase, which is the three-phase-boundary (TPB). The transmission current density of the battery is not only related to the partial pressure of the substance in the three-phase-boundary region, but also related to the activation polarization potential $^{[7]}$. SOFC is expensive to produce and difficult to test. In order to study the distribution of physical quantities inside the battery, many complex and effective mathematical equations and various numerical models have been developed. In addition to the impact of the microscopic configuration on the battery, the impact of changes in operating conditions in the experimental study of SOFC can also not be ignored. Because the operating conditions change, it will affect the electrochemical parameters and flow fields distribution of the battery, and affect the reaction rate at the electrode TPB, which

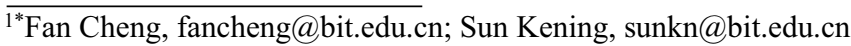

13120181317@bit.edu.cn corresponds to the response of the battery. The adjustment of operating parameters is an effective means to explore the optimal process conditions and reaction mechanism of SOFC ${ }^{[8]}$. Taking operating conditions and composition structure as variables, many researchers had conducted simulation studies and optimizations on SOFC, and have obtained rich research results. The research results of Abir Yahya $^{[9]}$ show that the discharge performance of the battery is related to the anode fuel gas partial pressure and the battery operating temperature. DiGiuseppe $\mathrm{G}^{[10]}$ studied the cathode reaction kinetics, and the results showed the importance of gas component partial pressure, and the oxygen partial pressure is strongly correlated with batteries discharge performance. Some research groups ${ }^{[11-}$ 14] found that changing operating conditions such as temperature and flow rate will also significantly affect the electrochemical performance of the battery. Numerical simulation can couple the mass transfer and reaction relationships of the three-phase-boundary to predict the output performance of the battery. It can provide reference for researchers.

In this paper, a three-dimensional anode-supported SOFC cell model based on Ni-YSZ//YSZ//LSM-YSZ is established. The model anode fuel gas is hydrogen. By combining relevant design parameters, the system studies the distribution of temperature fields, component concentration fields, current density and voltage inside the SOFC, and analyze the influence of operating parameters, including operating temperature and flow rate. 


\section{Model description}

\subsection{The geometric model}

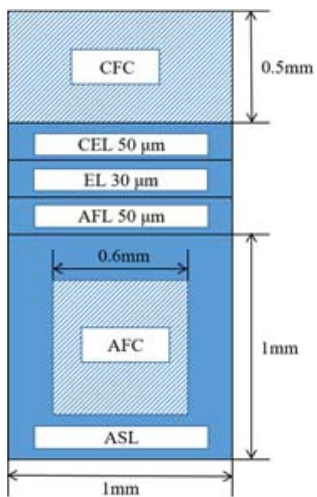

Fig. 1. Schematic cross section of flat tubular SOFC

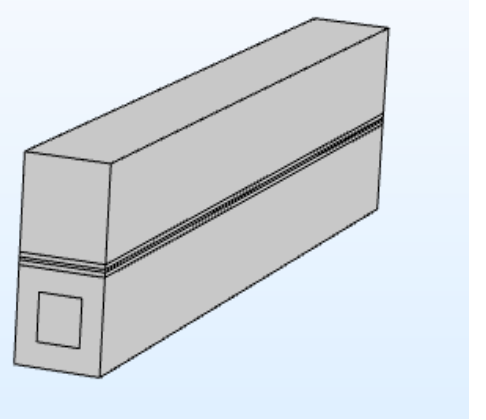

Fig. 2. Flat tubular SOFC geometric model diagram

The single cell model constructed in this paper consists of 5 parts (Figure 1), which are anode support layer (ASL), anode function layer (AFL), anode flow channel (AFC), and electrolyte layer (EL), and cathode electrode. Layer (CEL), cathode flow channel (CFC). The fuel gas flows in the flow channel of the anode support layer, and the air flows in the cathode flow channel.

\subsection{Model equation}

The internal reaction of SOFC mainly involves processes such as heat transfer, mass transfer, and electrochemical reaction. Among them, the electrochemical reaction occurs at the three-phase boundary of the electrode. Electromigration are transferred from the three-phaseboundary to the bulk phase. The reaction expressions of the cathode and anode TPB are shown in (1) and (2):

$$
\begin{gathered}
\mathrm{O}_{2}+2 \mathrm{e}^{-} \rightarrow 2 \mathrm{O}^{2-} \\
\mathrm{H}_{2}+\mathrm{O}^{2-}-2 \mathrm{e}^{-} \rightarrow \mathrm{H}_{2} \mathrm{O}
\end{gathered}
$$

The electrode reaction realizes the charge transfer, and the migration of electrons and oxygen ions is controlled by the electrode potential and the electrolyte potential; the electron current density and ion current density are shown by equations (3) and (4):

$$
\begin{aligned}
& \nabla \cdot i_{\text {ion }}=\nabla \cdot\left(-\sigma_{\text {ion }}^{\text {eff }} \nabla \eta_{\text {ion }}\right)=Q_{\text {ion }} \\
& \nabla \cdot i_{\text {ele }}=\nabla \cdot\left(-\sigma_{\text {ele }}^{\text {eff }} \nabla \eta_{\text {ele }}\right)=Q_{\text {ele }}
\end{aligned}
$$

The velocity fields distribution and pressure distribution of the model electrode are used for calculation through the free and porous medium flow fields. The gas flow in the flow channel adopts the continuous NavierStokes momentum conservation equation. In the battery model, due to the mass transfer caused by convection and diffusion, the concentration distribution of each participating reactant is solved by the concentrated substance diffusion fields. The governing equation is as follows:

$$
\begin{gathered}
\nabla\left(\rho \cdot u \cdot X_{i}\right)+\nabla J_{i}=M n_{i} r_{i} \\
\nabla\left(\rho \cdot u \cdot X_{i}\right)=\sum_{\mathrm{j}}-\rho D_{i, j}^{e f f} \cdot \nabla J_{i} \\
D_{i, j}^{e f f}=\varepsilon D_{i, j}
\end{gathered}
$$

The heat transfer process inside the battery consists of fluid heat transfer, porous medium heat transfer, solid heat transfer and heat radiation. The energy conservation equation is shown in equation (8).

$$
\rho C_{p} \frac{\partial T}{\partial \mathrm{L}}+\nabla \cdot\left(-\lambda_{\text {eff }} \nabla T\right)=Q_{\mathrm{r}}
$$

The electrode reaction kinetics of SOFC is expressed by the Butler-Volumer equation, which is applicable to both the cathode and anode of the fuel cell. The expression is shown in (9).

$$
i=i_{0}\left\{\frac{c_{\mathrm{Re}}}{c_{\mathrm{Re}}^{0}} \exp \left(\frac{\beta n F \eta_{a c t}}{R T}\right)-\frac{c_{\mathrm{Ox}}}{c_{\mathrm{Ox}}^{0}} \exp \left(\frac{-(1-\beta) n F \eta_{\mathrm{act}}}{R T}\right)\right\}
$$

When SOFC works, it is accompanied by three polarization phenomena, namely activation polarization, concentration polarization and ohmic polarization. Three polarization processes cause voltage loss and power loss.

$$
E_{\text {cell }}=E_{\text {Nernst }}-\eta_{\text {act }}-\eta_{\text {conc }}-\eta_{\text {ohm }}
$$

\section{Results and discussion}

\subsection{Model validation}

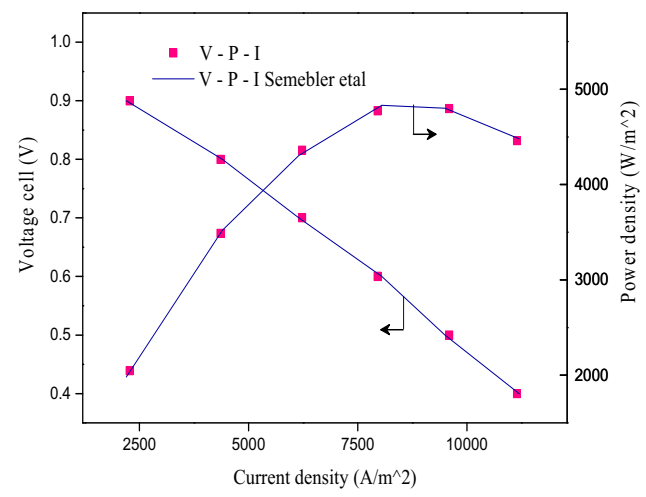

Fig. 3. Voltage and power density curves

It can be seen from Figure 3 that the battery voltage continuously decreases with the increase of current density, which is consistent with the test results of the actual discharge process. The maximum power density of the model SOFC is about $4700 \mathrm{~W} \cdot \mathrm{m}^{-2}$, and the overall 
discharge curve of the battery is in good agreement with the results reported by Sembler ${ }^{[15]}$, the model constructed in this paper is reliable and has reference significance.

\subsection{Current and voltage distribution}

The charge exchange reaction that occurs at the interface of the three-phase-boundary of the battery is not actually a uniform process, that is, the concentration distribution of ions and electrons varies with position. This is mainly due to factors such as conductive materials and battery configuration.

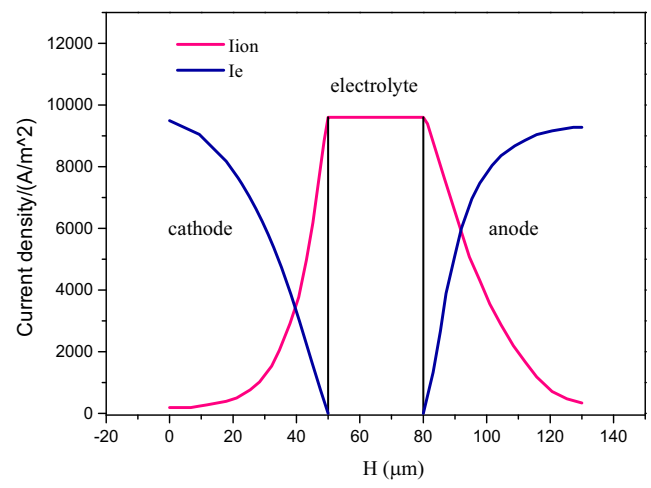

Fig. 4. Current density and voltage loss distribution of SOFC

Figure 4 shows the distribution curve of $I_{e}$ and $I_{i o n}$ along the electrode three-phase-boundary region when the battery voltage is $0.6 \mathrm{~V}$. Both $\mathrm{I}_{\mathrm{e}}$ and $\mathrm{I}_{\text {ion }}$ fall one after another. The electrons transmitted from the battery boundary and the oxygen transported through the cathode porous structure underwent a catalytic reaction on the surface of LSM/YSZ to generate oxygen ions. This charge exchange reaction continues to deepen along the thickness of the battery, showing a decrease in $\mathrm{I}_{\mathrm{e}}$ and the law of increase of $\mathrm{I}_{\text {ion }}$ : At the boundary between the cathode and the electrolyte layer, since the electrolyte layer is only a good conductor of oxygen ions, blocking the transmission of gas on the cathode side and blocking the flow of electrons, $I_{\mathrm{e}}$ is 0 at the interface between the cathode and the electrolyte layer. Hydrogen and oxygen ions conducted via YSZ undergo a reduction reaction in the anode three-phase-boundary region, releasing electrons, and the electrons are transferred to the outside along the anode's conductive network. This process along the thickness of the anode is a process in which $\mathrm{I}_{\text {ion }}$ decreases and $\mathrm{I}_{\mathrm{e}}$ increases.

As shown in Figure 5, the overpotential loss and the current density change along the length of the battery are consistent, and both show a decreasing trend. This is mainly due to the limited diffusion and mass transfer of materials and the effect of electrochemical reactions on the consumption of reactants. The partial pressure of the fuel gas decreases along the flow channel, which increases the concentration polarization loss of the battery and affects the electrochemical reaction rate. The decrease gradient of overpotential loss and current density both gradually slow down along the fluid flow direction. The SOFC temperature shows an upward trend along the fluid flow direction, which gradually reduces the activation overpotential loss. As the cathode oxygen concentration polarization loss increases at the outlet, the overpotential drop is slowed down. Under this operating condition, the negative effect of the change of reactant concentration on the polarization loss is stronger than the positive effect of high temperature on the electrochemical reaction.

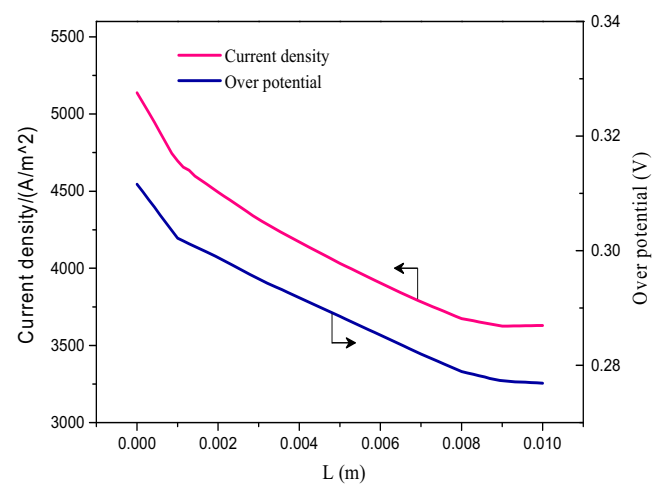

Fig. 5. Distribution of output current density and voltage loss along the direction of fluid flow

In Figure 6, in the polarization curves of the inlet and outlet, the law of voltage loss is basically the same. The most important voltage loss along the thickness of the SOFC occurs in the cathode three-phase-boundary region, that is, the polarization loss of the cathode accounts for the main part, accounting for about $45 \%$. Due to the sufficient amount of hydrogen on the anode side and uniform distribution, the anode polarization loss is minimal. At the same time, the ohmic loss of the electrolyte layer cannot be ignored, and its proportion is about 30\%. The overpotential gap between the outlet and the inlet sides is quite different in the cathode three-phase-boundary region. This is because the temperature at the outlet is higher, which reduces the activation overpotential loss and ohmic loss. However, the partial pressure of the reactants on the outlet sides decreases and the concentration polarization increases. The combined effect of the three polarizations makes the difference in pressure drop loss between the inlet and outlet sides obvious.

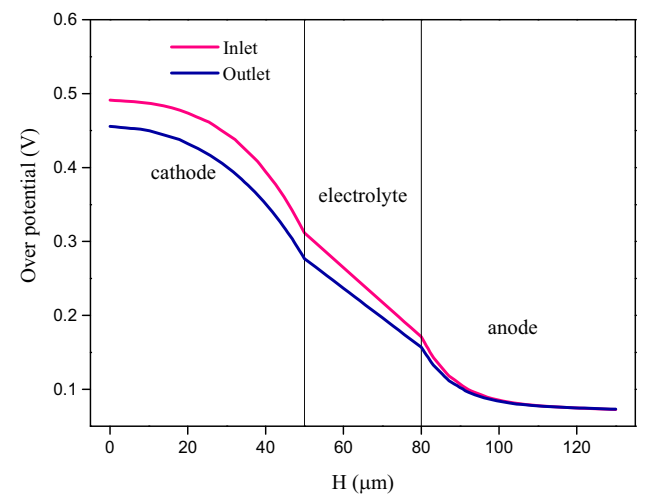

Fig. 6. Voltage loss distribution

\subsection{The effect of temperature on battery performance}

The air and fuel gas flow rates are both $20 \mathrm{~mL} / \mathrm{min}$, and the SOFC performance at operating temperatures of $973 \mathrm{~K}$, $1073 \mathrm{~K}$ and $1173 \mathrm{~K}$ are compared. The results are as follows. 


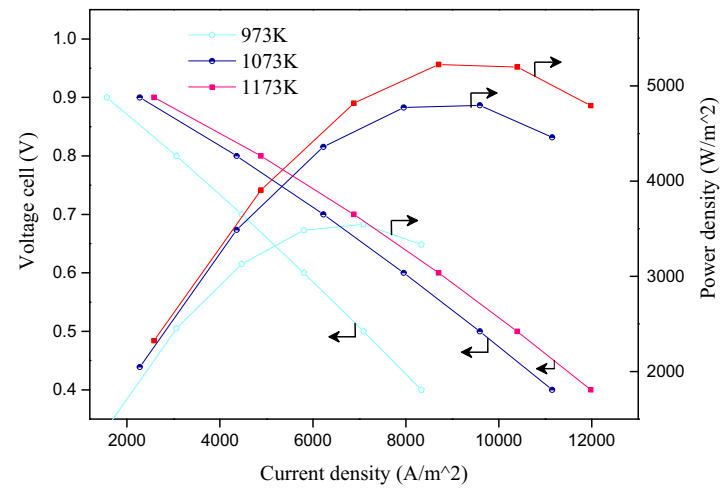

Fig. 7. V-I-P curve at different operating temperatures

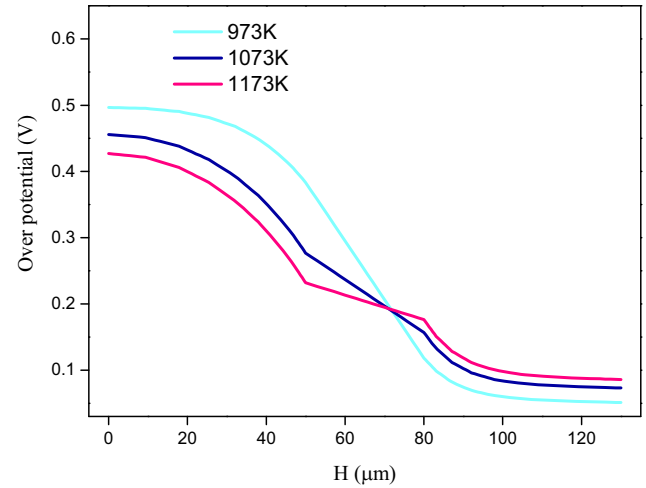

Fig. 8. Overpotential distribution along the height of the battery at the inlet of SOFC

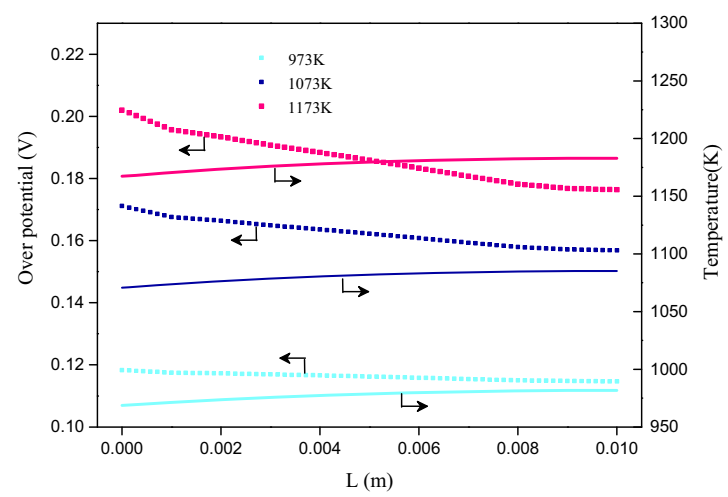

Fig. 9. Overpotential distribution of SOFC along the length of the battery

The electrochemical performance of the battery is gradually improved with the increase of temperature, and the gradient of the influence of cooling on battery performance is higher than that of heating. It can be seen from Figure 8 that the increase in temperature reduces the activation energy barrier of the electrochemical reaction and the activation overpotential loss. Because the electrolyte conductivity is positively correlated with temperature, the ohmic loss of the electrolyte is significantly reduced; when the temperature rises, the gas diffusion rate is accelerated, and more reactive gas diffuses to the three-phase-boundary to participate in the electrochemical reaction, and the electrochemical reaction rate is accelerated, resulting in a decrease in the loss of concentration over-potential. Along the length of the battery, the overpotential loss is consistent with the temperature gradient of the battery as a whole.

\subsection{The influence of air flow on battery performance}

Air is the fuel gas for the electrochemical reaction on the cathode side. Due to the flow and heat exchange of the gas, the excess air has a cooling effect on the battery. Set the model operating temperature to $1073 \mathrm{~K}$, and the hydrogen flow rate on the anode side to $20 \mathrm{~mL} / \mathrm{min}$. Study the influence of air flow rate on battery performance.

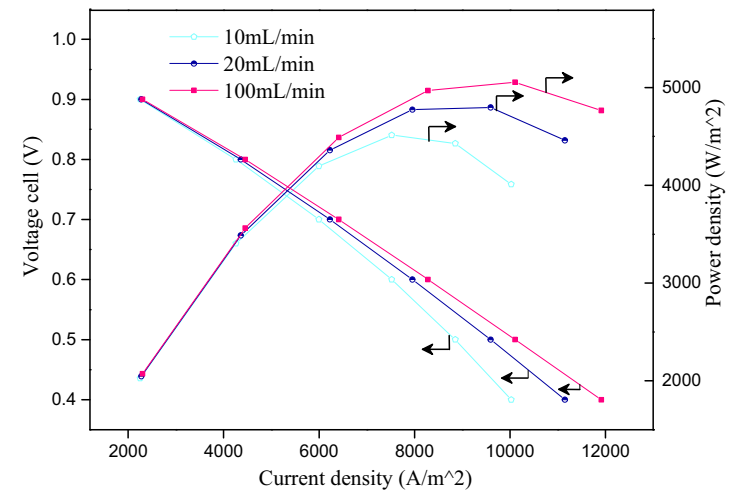

Fig. 10. V-I-P curve at different air flow

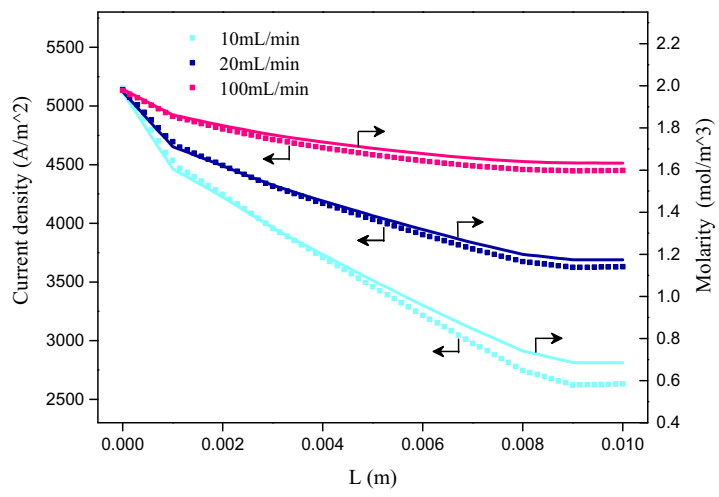

Fig. 11. Distribution of current density and oxygen concentration along the length of the battery

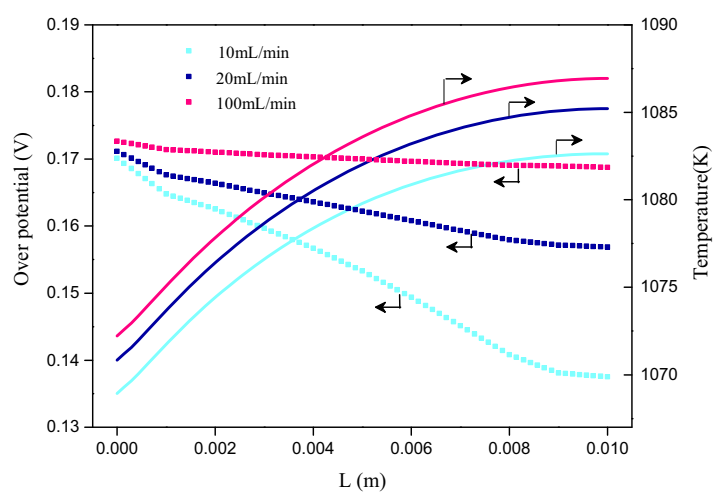

Fig. 12. Distribution of voltage loss and temperature along the length of the battery

As the air flow increases, the oxygen increases, the fuel gas concentration gradient decreases, the reaction rate of the three-phase-boundary increases, and the SOFC 
performance is improved. However, the improvement of battery performance and the increase in air flow are not a single linear relationship. When the current density is low, increasing the air flow has a greater impact on the activation polarization, and has a small impact on the battery discharge performance; When the current density is high, based on the dominance of cathode polarization, the battery concentration polarization is significant. Increasing the air flow can increase the diffusion rate of the gas to and from the reaction activation site, improve the concentration polarization phenomenon, and improve the performance of the SOFC. It can be seen from Figure 11 that along the length of the battery, the current density and the oxygen concentration have the same changing trend. As the fuel gas flow rate increases, the current density increases. The greater the air flow, the shorter the stay in the SOFC, and the lower the fuel utilization rate.

\section{Conclusion}

Based on the basic principles of electrochemistry, this paper established a physical model of an anode-supported flat tube solid oxide fuel cell, discussed the influence of design parameters and operating parameters on the threephase-boundary of the battery, and obtained the following conclusions:

(1) Increasing the operating temperature will reduce the polarization loss and significantly improve the discharge performance of the battery;

(2) Increasing the air flow can enhance the mass transfer process, reduce the concentration polarization loss, and improve the discharge performance of the battery. When the current density is low, the flow rate has less impact on the battery performance. When the current density is high, the demand for oxygen increases due to the electrochemical reaction rate, and the effect of increasing the flow rate is obvious.

These conclusions are expected to guide the structural design, material development and selection of operating parameters of fuel cells, and have a reference role for the engineering application of fuel cells.

\section{References}

1. Si X, Cao J, Liu S, et al. (2018) Fabrication of 3D $\mathrm{Ni}$ nanosheet array on Crofer22APU interconnect and NiO-YSZ anode support to sinter with smallsize Ag nanoparticles for low temperature sealing SOFCs. International Journal of Hydrogen Energy, 43(5): 2977-2989.

2. Nerat M. (2017) Modeling and analysis of shortperiod transIent response of a single, planar, anode supported, solid oxide fuel cell during load variations. Energy, 138: 728-738.

3. XIe J, Xu M Y, Ban S, et al. (2019) Simulation analysis of multi-physics coupling SOFC fueled nature gas in the way of internal reforming and external reforming. CIeSC Journal, 70(1): 214-226.
4. Bora T, Cigdem T, Yuksel K, et al. (2016) A revIew on cell/stack designs for high performance solid oxide fuel cells. Renewable and Sustainable Energy RevIews, 56: 1101-1121.

5. Su S, Zhang W, Wu J, et al. (2017) Effect of component thickness and anode composition on the residual stress of micro-tubular solid oxide fuel cell.International Journal of Electrochemistry, 12: 9121-9130.

6. Liu Y, Tang Y, Ding J, et al. (2012) Electrochemical performance of cone-shaped anode-supported segmented-in-serIes SOFCs fabricated by gelcasting technique. International Journal of Hydrogen Energy, 37(1): 921-925.

7. Lee S, Kim H, Yoon K J, et al. (2016) The effect of fuel utilization on heat and mass transfer within solid oxide fuel cells examined by threedimensional numerical simulations. International Journal of Heat and Mass Transfer, 97: 77-93.

8. Ramirez-Minguela J J, Mendoza-Miranda J M, Rodriguez-Munoz J L, et al. (2018) Entropy generation analysis of a solid oxide fuel cell by computational fluid dynamics: Influence of electrochemical model and its parameters. Thermal ScIence, 22(1): 577-589.

9. Yahya A, Ferrero D, Dhahri h, et al. (2018) Electrochemical performance of solid oxide fuel cell: Experimental study and calibrated model[J]. Energy, 142: 932-943.

10. Digiuseppe G, Sun L. (2011) Electrochemical performance of a solid oxide fuel cell with an LSCF cathode under different oxygen concentrations. International Journal of Hydrogen Energy, 36(8): 5076-5087.

11. Khazaee I, Rava A. (2017) Numerical simulation of the performance of solid oxide fuel cell with different flow channel geometrIes. Energy, 119(15): 235-244.

12. Ilbas M, Kumuk B. (2018) Numerical modelling of a cathode-supported solid oxide fuel cell in comparison with an electrolyte-supported model. Journal Energy Institute, 92(3): 682-692.

13. Ghorbani B, Vijayaraghavan K. (2018) 3D and simpliffed pseudo-2D modeling of single cell of a high temperature solid oxide fuel cell to be used for online control strategIes. International Journal of Hydrogen Energy, 43(20): 9733-9748.

14. Takino K, Tachikawa Y, Mori K, et al. (2020) Simulation of SOFC performance using a modifled exchange current density for pre-reformed methane-based fuels. International Journal of Hydrogen Energy, 45(11): 6912-6925.

15. William J S, Kumar S. (2011) Modification of results from computational-fluid-dynamics simulations of single-cell solid oxide fuel cells to estimate multicell stack performance. Journal of Fuel Cell ScIence \& Technology, 8(2): 1008-1017. 